TRABAJOS ORIGINALES

\title{
Efecto de Maytenus macrocarpa "Chuchuhuasi" en el sistema reproductor masculino del ratón (Mus musculus)
}

\author{
Effect of Maytenus macrocarpa "Chuchuhuasi" in the male system reproductive of mouse \\ (Mus musculus)
}

\author{
Láyonal G. Acosta ${ }^{1,2 *}$, Jonathan Vásquez¹, Víctor Núñez ${ }^{1}$, José Pino ${ }^{1}$, Betty Shiga ${ }^{1}$
}

1 Laboratorio de Reproducción y Biología del Desarrollo, Instituto de Investigación de Ciencias Biológicas Antonio Raimondi, Facultad de Ciencias Biológicas Universidad Nacional Mayor de San Marcos (UNMSM). Lima - Perú 2 BIOGENETIC LAB SAC. Chiclayo - Perú.

Autor para correspondencia:

Biólogo Genetista. Láyonal Germán Acosta Campos. BIOGENETIC LAB SAC. Chiclayo - Perú. Laboratorio de Reproducción y Biología del Desarrollo, Facultad de Ciencias Biológicas, Universidad Nacional Mayor de San Marcos, Lima, Perú. Casilla 11-058, Lima 11, Perú. Tel.: +51 6197000 - 1529; fax: +51 $6197000-1509$.

Email Láyonal Acosta: acostaclg@gmail.com

Citación:

Acosta L.G., J. Vásquez, V. Núñez., J. Pino, B. Shiga. 2014. Efecto de Maytenus macrocarpa "Chuchuhuasi" en el sistema reproductor masculino del ratón (Mus musculus). Rev. peru. biol. 20(3): 223 - 226 (Marzo 2014)

\section{Resumen}

Maytenus macrocarpa (Chuchuhuasi) es un árbol nativo de la región Amazónica del Perú utilizado como planta medicinal para el tratamiento de muchas enfermedades, sin embargo su efecto sobre el sistema reproductor masculino aún no ha sido elucidado. El objetivo del presente estudio fue evaluar el efecto del extracto acuoso de $M$. macrocarpa en dosis diarias durante 7 días sobre los parámetros reproductivos de ratones machos. Se utilizó machos maduros de la cepa C57BL divididos en 2 grupos $(n=10)$, Grupo Control (C): $\mathrm{NaCl} 0.9 \%$ y Grupo Tratamiento (T): Extracto acuoso de Chuchuhuasi, ambos suministrado diariamente vía sonda esofágica y al octavo día fueron eutanizados. Se registraron el peso corporal y de los órganos reproductivos: testículo, epidídimo y conducto deferente, se evaluaron la concentración, movilidad y morfología espermática. En nuestros datos observamos diferencias significativas (Prueba t- Student $\mathrm{P}=0.05$ ) en el peso de la cabeza y cuerpo del epidídimo (C: $19.25 \pm 1.1$ vs T: $21.26 \pm 2.0$ ), el peso del conducto deferente (C: $10.62 \pm 0.7$ vs T: $11.75 \pm 0.5$ ), movilidad espermática progresiva (C: $42.16 \pm 5.2$ vs T: $25.82 \pm 8.4$ ) e Inmóviles (C: $36.05 \pm 4.9$ vs T: $48.51 \pm 7.2$ ). No se observaron diferencias en el recuento espermático entre ambos grupos. La morfología espermática normal disminuyó con la ingesta de M. macrocarpa (Prueba t-Student $p<0.05)(C: 39.72 \pm 1.3$ vs T: $30.78 \pm 4.9)$. Concluimos que el extracto acuoso de $M$. macrocarpa presenta un efecto negativo en el sistema reproductivo masculino de ratones.

Palabras claves: Maytenus macrocarpa, Chuchuhuasi, ratón, calidad espermática, infertilidad.

\section{Abstract}

Maytenus macrocarpa (chuchuhuasi) is native tree of the Peruvian Amazon used as traditional medicine for the treatment of many diseases, but its effect on the male reproductive system has not yet been elucidated. The aim of this study is evaluate the effect of aqueous extract of M. macrocarpa in daily doses for 7 days on reproductive parameters of male mice. We used C57BL mature male mice divided into 2 groups $(n=10)$, Control Group (C): $0.9 \% \mathrm{NaCl}$ and Treatment group $(\mathrm{T})$ : Aqueous extract of Chuchuhuasi, both supplied daily via oral gavages. At the eight day of treatment the mice were euthanized. The weight of the body and reproductive organs: testis, epididymis and vas deferens, were registered. Concentration, motility and sperm morphology were evaluated. The results showed significantly differences (t- Student test $P<0.05)$ in the weight of the head and body epididymis $(C: 19.25 \pm 1.1$ vs $T: 21.26 \pm 2.0)$, vas deferens (C: $10.61 \pm 0.7$ vs T: $11.75 \pm 0.5)$, progressive sperm motility (C: $42.16 \pm 5.2$ vs T: $25.82 \pm 8.4$ ) and immobile sperm (C: $36.05 \pm 4.9$ vs T: $48.51 \pm 7.2$ ). No difference in sperm count was observed. The sperm normal morphology diminished with ingest of $M$. macrocarpa (tStudent test $p<0.05)(C: 39.72 \pm 1.3$ vs T: $30.78 \pm 4.9$ ). We conclude that the aqueous extract of chuchuhuasi, has a negative effect on the male reproductive system of mice.

Keywords: Maytenus macrocarpa, Chuchuhuasi, mouse, sperm quality, infertility.

\section{Introducción}

Las plantas y sus derivados han sido usados como alimentos y fuente importante de principios activos para el tratamiento de distintas enfermedades. El potencial terapéutico de la mayoría de estas plantas esta basado en sus propiedades anticancerígenas, antidiabéticas, hepatoprotectivas, cardioprotectivas, antipasmódicas, analgésicas y varias otras propiedades farmacológicas (D'Cruz et al. 2010). 
Sin embargo, algunas plantas pueden afectar negativamente distintas funciones fisiológicas normales, entre ellas la función reproductiva masculina, cuyos efectos adversos han sido atribuidos principalmente a propiedades antiespermatogénicas y antiesteroidogénicas de uno o más compuestos activos (Lohiya et al. 2002, Ashok \& Meenakshi 2004, Gupta et al. 2004). En el Perú, aproximadamente 1400 especies de plantas son utilizadas con fines medicinales, cuyo uso popular se conoce desde épocas prehispánicas.

Los extractos de las plantas de la familia Celastráceas han sido utilizados por siglos como insecticidas y para el tratamiento de complicaciones estomacales, fiebre, reumatismo y cáncer (Mejía \& Reng 1995). El género Maytenus es el más abundante e importante de la familia Celastráceas (Santos et al. 2007).

Se ha demostrado la actividad biológica de varias especies de Maytenus: $M$. ilicifolia como anticonceptivo, antiinflamatorio y antioxidante (Jorge et al. 2004, Vellosa et al. 2006); M. krukovii presenta actividad antioxidante, antimutagénica y antimicrobiana (Bruni et al. 2006); $M$. aquifolium, propiedades analgésicas (Gonzalez et al. 2001); M. senegalensis posee actividad antibacteriana, antiviral, antitumoral (Gessler et al. 1995, Otake et al. 1995, Matu \& Van Staden 2003) y M. macrocarpa actúa como citotóxico de células cancerígenas (Chavez et al. 2010) y antimicrobiano (Kloucek et al. 2007).

El género Maytenus contiene compuestos activos como los maitansinoides que ejercen actividad insecticida (Madrigal et al. 1985), triterpenoquinonas y dímeros triterpénicos con actividad antimicrobiana (González et al. 1996a) y nortriterpeno metilénquinonas con funciones antimicrobianas (González et al. 1996b).

Existe poca información de los efectos de las plantas de Maytenus sobre el sistema reproductor de mamíferos. Montanari et al. (1998) proporcionaron dosis diarias de $800 \mathrm{mg} / \mathrm{kg}$ peso corporal (pc) de $M$. ilicifolia a ratones machos por 20 días y no encontraron efecto alguno sobre la espermatogénesis, sin embargo Montanari y Bevilacqua (2002) en dosis diarias de $1000 \mathrm{mg} / \mathrm{kg}$ pc concluyeron que interfiere con la implantación embrionaria en ratones.

Maytenus macrocarpa (Chuchuhuasi) es una planta nativa de la región Amazónica del Perú, y es usado como planta medicinal desde la época del antiguo Perú. Previos estudios han reportado el aislamiento de triterpenos dammarane y triterpenos friedelane a partir de la corteza del tallo (Chavez et al. 1997, Chávez et al. 1998), poliésteres sesquiterpénicos de las hojas (Chavez et al. 1999) y nortriterpenos macrocarpines A-D de las raíces de $M$. macrocarpa (Chavez et al. 2000). Su corteza es utilizada generalmente como aguardiente para el tratamiento de reumatismo, influenza, enfermedades gastrointestinales y como agente antitumoral (Dhingra et al. 2000), sin embargo su efecto sobre el sistema reproductor masculino aún no ha sido elucidado.

El objetivo del presente estudio fue evaluar el efecto de la ingesta de extracto acuoso de $M$. macrocarpa $(1000 \mathrm{mg} / \mathrm{kg} \mathrm{pc})$ durante 7 días sobre los parámetros reproductivos de ratones machos.

\section{Material y métodos}

Animales y condiciones éticas.- Los experimentos se llevaron a cabo con ratones (Mus musculus) machos $(\mathrm{n}=20)$ de 8 a 10 semanas de edad de la cepa C57BL. Los animales fueron adquiridos en el Bioterio de la Universidad Peruana Cayetano Heredia (UPCH), Lima, Perú y aclimatados durante una semana previa a los ensayos en el Bioterio de la Facultad de Ciencias Biológicas de la Universidad Nacional Mayor de San Marcos (UNMSM), Lima, Perú, siguiendo lo indicado en la Guía de manejo y cuidado de animales de laboratorio: ratón del Instituto Nacional de Salud (Fuentes 2008).

Los animales fueron mantenidos bajo condiciones estándar de bioterio con clima controlado $\left(23 \pm 5^{\circ} \mathrm{C}, 90 \pm 5 \%\right.$ humedad $)$ fotoperiodo de $14 \mathrm{~h}$ luz: $10 \mathrm{~h}$ oscuridad, alimentados con dieta balanceada para animales (Bedoce) y agua ad libitum un total de 15 días mientras duró la aclimatación y los ensayos.

Los animales fueron eutanizados por dislocación cervical siguiendo los lineamientos del Consejo Nacional de Investigación para el cuidado y uso de animales de laboratorio (NRC, 1996).

Extracto acuoso.- Maytenus macrocarpa fue obtenido de PromoAgro Export CC (Lima, Perú; voucher botánico Co-V060-013). El extracto acuoso fue preparado del material seco y pulverizado de la corteza. Cien gramos de $M$. macrocarpa fue hervido por 30 minutos en un litro de agua y se dejo enfriar durante toda la noche a temperatura ambiente. Al siguiente día el sobrenadante fue filtrado dos veces usando papel filtro estéril Whatman (40 $\mu$ m y $20 \mu \mathrm{m}$, respectivamente). El extracto resultante fue alicuotado en microtubos de $1.5 \mathrm{~mL}$ y almacenados a $4{ }^{\circ} \mathrm{C}$ hasta su uso.

Diseño experimental.- Los ratones fueron divididos en dos grupos; el grupo Tratamiento $(\mathrm{T})(\mathrm{n}=10)$, se le administró el extracto acuoso de $M$. macrocarpa $(1000 \mathrm{mg} / \mathrm{kg} \mathrm{pc})$ y al grupo control (C) $(\mathrm{n}=10)$ solución salina $\mathrm{NaCl} 0.9 \%$. Al inicio del tratamiento se registro su peso corporal. El extracto acuoso de $M$. macrocarpa y la solución salina fueron administrados usando una sonda esofágica $\mathrm{N}^{\circ} 18$ (Fisher Scientific, Pittsburgh, PA, USA). Al octavo día después del tratamiento se registró el peso corporal final y los animales fueron eutanizados por dislocación cervical. Se pesaron: testículos, epidídimos y conductos deferentes. Se obtuvieron espermatozoides de la cola del epidídimo para registrar la movilidad, concentración y morfología espermática.

Obtención de epidídimos y espermatozoides.- Cada uno de los epidídimos obtenidos fueron lavados con buffer fosfato salino (PBS pH 7.4) a $37^{\circ} \mathrm{C}$, se realizaron varios cortes en la cola del epidídimo y luego los espermatozoides se liberaron permaneciendo durante 10 minutos en $0.5 \mathrm{~mL}$ de medio de cultivo Flushing (MediCult ${ }^{\bullet}$, Copenhagen, Dinamarca), el contenido espermático se recuperó en su totalidad en tubos de polipropileno de $1.5 \mathrm{~mL}$ (Axygen Scientific).

Evaluación de la movilidad espermática.- Muestra espermática fue colocada sobre una lámina portaobjetos a $37^{\circ} \mathrm{C}$ y observada a 400X en un microscopio óptico de contraste de fases (Carl Zeiss Jena). Este procedimiento fue repetido dos veces, los resultados presentados son el promedio de ambas evaluaciones, contabilizándose al menos 100 espermatozoides. Se consideró que un espermatozoide presentaba Movilidad Progresiva (MP), cuando presentaba desplazamiento, Movilidad No Progresiva (MNP) a aquellos que no se desplazaban y sólo tenían un movimiento in situ e Inmóviles (IM) los que no presentaban ningún tipo de movimiento (WHO, 2010). 
Tabla 1. Efecto del extracto acuoso del "Chuchuhuasi" Maytenus macrocarpa sobre el peso corporal, peso de testículo, epidídimo y conducto deferente entre el grupo Control $(\mathrm{C})$ y Tratamiento $(\mathrm{T})$. Los valores son expresados como media $\pm \mathrm{DS}$. C=Control peso corporal, $\mathrm{T}=\mathrm{Tratamiento}$ peso corporal.

\begin{tabular}{lccc}
\hline & Control (NaCl 0.9\%) & Chuchuhuasi (1000mg/kg pc) & $P$ \\
\hline Ratones analizados (n) & 10 & 10 & \\
Peso corporal inicial (g) & $21.40 \pm 0.5$ & $27.30 \pm 1.6$ & $\mathrm{C}=0.020$ \\
Peso corporal final (g) & $23.35 \pm 1.2$ & $26.62 \pm 1.9$ & $\mathrm{~T}=0.248$ \\
Peso testículo (mg) & $72.99 \pm 5.5$ & $78.02 \pm 8.2$ & 0.118 \\
Peso de cabeza + cuerpo del epidídimo (mg) & $19.25 \pm 1.1$ & $21.26 \pm 2.0$ & 0.034 \\
Peso de la cola del epidídimo (mg) & $143.1 \pm 2.2$ & $143.3 \pm 1.7$ & 0.980 \\
Peso del conducto deferente (mg) & $10.62 \pm 0.7$ & $11.75 \pm 0.5$ & 0.003 \\
\hline
\end{tabular}

Medición de la concentración espermática.- La concentración espermática se midió usando una dilución 1:20, 10 $\mu \mathrm{L}$ de la muestra se diluyó con $190 \mu \mathrm{L}$ solución de fijación (WHO, 2010) posteriormente se colocó $10 \mu \mathrm{L}$ de la dilución en la cámara de Neubauer, y se contaron los espermatozoides dentro de los campos definidos en la cámara, mediante el uso de un microscopio óptico de contraste de fases 400X, la cantidad de espermatozoides observados se multiplicó por el factor correspondiente $\left(10^{6}\right)$. La concentración de espermatozoides fue expresada en millones $/ \mathrm{mL}$.

Evaluación de la morfología espermática.- Los espermatozoides fueron expandidos mediante un frotis sobre láminas portaobjetos, se dejó secar a temperatura ambiente, se fijaron con paraformaldehído por 20 minutos y se colorearon con Eosina-Y al 5\%. Se analizaron 250 espermatozoides por espécimen bajo un microscopio óptico (Carl Zeiss Jena, Amplival) de campo claro con objetivo de inmersión (1000X). Se consideró como anormal, aquel espermatozoide que presentaba alteraciones morfológicas en cabeza, pieza intermedia, cola o en presencia de más de una alteración. Espermatozoides con gota citoplasmática fueron considerados inmaduros.

Análisis estadísticos.- Los análisis estadísticos fueron realizados con el programa SPSS v21 para Windows. Los datos cumplieron las premisas de normalidad y homogeneidad de varianza (Prueba de Levene) por lo cual todos los resultados se analizaron mediante la prueba de T-student. El nivel de significancia establecido fue $P<0.05$.

\section{Resultados}

Peso corporal y órganos reproductivos.- En el grupo control se observó diferencias significativas en el peso corporal inicial y final $(P=0.020)$. Así mismo se observó diferencias significativas entre el grupo control y tratamiento en el peso de la cabeza y el cuerpo del epidídimo $(P=0.034)$ y el peso del conducto deferente $(P=0.003)$. Los resultados se resumen en la Tabla 1 .

Movilidad y concentración espermática.- No se observó diferencias significativas en la concentración espermática entre ambos grupos (Tabla 2). Respecto a la movilidad espermática se observa diferencias significativas $(P<0.05)$ en la Movilidad Progresiva (C: $42.16 \pm 5.2$ vs T: $25.82 \pm 8.4$ ) e Inmóviles (C: $36.05 \pm 4.9$ vs T: $48.51 \pm 7.2)$. No se encontró diferencias significativas en la Movilidad No Progresiva (C: $25.38 \pm 4.0$ vs T: 29.16 \pm 5.5 ) (Figura 1).

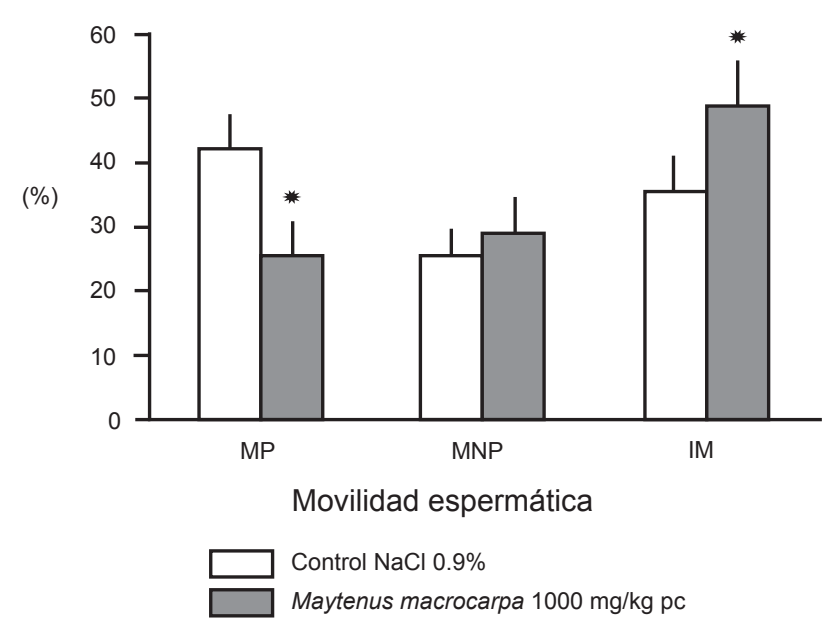

Figura 1. Efecto del extracto acuoso del "Chuchuhuasi” Maytenus macrocarpa sobre la movilidad espermática. MP: Movilidad progresiva; MNP: Movilidad no progresiva; IM: Inmovilidad; pc: peso corporal. Los valores son expresados como media $\pm \mathrm{SD}$. ${ }^{*} P<0.05$ en comparación con el grupo control.

Tabla 2. Efecto del extracto acuoso del Chuchuhuasi Maytenus macrocarpa sobre la concentración y morfología espermática. PI: Pieza intermedia, pc: peso corporal. Los valores son expresados como media \pm SD. ${ }^{*} \mathrm{P}<0.05$ en comparación con el grupo control.

\begin{tabular}{lcc}
\hline & Control NaCl 0.9\% & Chuchuhuasi 1000 mg/kg pc \\
\hline$N^{\circ}$ ratones analizados & 10 & 10 \\
Concentración espermática $\left(\times 10^{6} / \mathrm{mL}\right)$ & $14,40 \pm 3.3$ & $15,04 \pm 2.2$ \\
Normales & $39,72 \pm 1,3$ & $30,78 \pm 4,9^{*}$ \\
Anormalidad de la cabeza & $23,91 \pm 5,9$ & $26,74 \pm 3,7$ \\
Anormalidad de la PI & $50,59 \pm 6,5$ & $49,88 \pm 10,9$ \\
Anormalidad de la cola & $31.73 \pm 9,1$ & $30,70 \pm 5,9$ \\
Gota citoplasmática & $18,51 \pm 8,6$ & $23,41 \pm 9,2$ \\
\hline
\end{tabular}


Morfología.- Las evaluaciones morfológicas mostraron diferencias significativas (prueba t-student, $P<0.05$ ) en el porcentaje de espermatozoides normales entre el grupo $\mathrm{T}$ y el grupo $\mathrm{C}$ (Tabla 2). Al evaluar la distribución de anormalidades (cantidad neta de ocurrencias por cada anormalidad observada) no se observó diferencias significativas entre ambos grupos (Tabla 2).

\section{Discusión}

El presente estudio se realizó para evaluar si el extracto acuoso de $M$. macrocarpa (1000 mg/kg pc) produce toxicidad sobre el sistema reproductor masculino.

No se observó diferencias significativas en el peso corporal de los ratones suministrados con $M$. macrocarpa "Chuchuhuasi" (Tabla 1) sugiriendo que el extracto acuoso no causaría toxicidad sistémica en el individuo a la concentración suministrada. M. macrocarpa "Chuchuhuasi" presenta fitoelementos como triterpenos cuya actividad antiinflamatoria (Safavhi y Sailer 1997, Nikiema et al., 2001, Costa et al. 2003) se ha asociado con la inhibición de la ciclooxigenasa 2 (COX-2) (Medeiros et al. 2007), molécula relacionada a la síntesis de prostaglandina, moléculas importantes en la movilidad de los espermatozoides (Balaji et al. 2007a, b). Además, contiene alcaloides que inhiben la producción de proteína quinasa C (PKC) (Bradshaw et al. 1993) asociada también a la movilidad espermática (White et al. 2007), alguno de estos compuestos podría estar disminuyendo la movilidad espermática reportada en esta investigación.

Los resultados con respecto a la morfología espermática, sugieren que $M$. macrocarpa "Chuchuhuasi" afectaría a la espermatogénesis en el estadio de espermátide tardía, al final de la espermiogénesis, posiblemente alterando la condensación de la cromatina, ocasionando un aumento de la morfología anormal reportado en este estudio. Basados en los resultados del presente estudio concluimos que el extracto acuoso de $M$. macrocarpa "Chuchuhuasi" en dosis de $1000 \mathrm{mg} / \mathrm{kg}$ pc, presenta un efecto negativo en el sistema reproductivo masculino de ratones.

\section{Literatura citada}

Ashok P. \& B.Meenakshi. 2004. Contraceptive effect of Curcuma longa (L.) in male albino rat. Asian J. Androl. 6:71-74.

Balaji T., M. Ramanathan \& V.P.Menon. 2007a. Localization of cyclooxygenase-2 in mice vas deferens and its effects on fertility upon suppression using nimesulide - a preferential cyclooxygenase-2inhibitor. Toxicology. 234:135-144.

Balaji T., M. Ramanathan, V.P. Menon. 2007b. Localization of cyclooxygenase-2 in mice testis and assessment of its possible through suppressing its expression usingnimesulide-a preferential cyclooxygenase-2 inhibitor.Prostaglandins Leukot.Essent. Fatty Acids. 76:341-348.

Bradshaw D., C.H. Hill, J.S. Nixon, et al. 1993. Therapeutic potential of protein kinase C inhibitors. Agents and Actions. 38:135-147.

Bruni R., D. Rossi, M. Muzzoli, et al. 2006. Antimutagenic, antioxidant and antimicrobial properties of Maytenus krukovii bark. Fitoterapia. 77:538-545.

Chavez H, A.E. Estevez-Braun, A.G. Ravelo, et al. 1997. First examples of dammarane triterpenes isolated from Celastraceae. Tetrahedron. 53:6465-6472.

Chavez H., A.E. Estevez-Braun, A.G. Ravelo, et al. 1998. Friedelane triterpenoids from Maytenus macrocarpa. J. Nat. Prod. 61:82-85.

Chavez H., N. Callo, A.E. Estevez-Braun, et al. 1999. Sesquiterpene polyolesters from the leaves of Maytenus macrocarpa. J. Nat. Prod. 62:1576-1577.

Chavez H., G. Rodriguez, A.E. Estevez-Braun, et al. 2000. Macrocarpins A-D, new cytotoxic nor-triterpenes from Maytenus macrocarpa. Bioorg Med Chem Lett. 10:759-762.
Costa V.B., C.S. Coube, B.G. Marinho, et al. 2003. Antiinflammatory and analgesic activity of Bouche afluminensis. Fitoterapia. 74:364-371.

D'Cruz S.C., S. Vaithinathan, R. Jubendradass, et al. 2010. Effects of plants and plant products on the testis. Asian J. Androl. 12:468-479.

Dhingra V., K.V. Rao \& M. Narasu. 2000. Current status of artemisinin and its derivatives as antimalarial drugs. Life Sciences. 66:279-300.

Fuentes F.M., R.A. Mendoza, A.L. Rosales \& R.A. Cisneros. 2008. Guía de manejo y cuidado de animales de laboratorio: ratón. Instituto Nacional de Salud (Perú). Ministerio de Salud, Instituto Nacional de Salud. Lima. Pp 52.

Gessler M.C., M. Tanner, J. Choll, et al. 1995. Tanzanian medicinal-plants used traditionally for the treatment of malaria - in vivo antimalarial and in vitro cytotoxic activities. Phytother. Res. 9:504-508.

González A.G., N. Alvarenga, A. Ravelo, et al. 1996a. Scutione, a new bioactive norquinone- methide triterpene from Maytenus scutioides (Celastraceae). Biorg. Med. Chem. 4:815-820.

González A.G., N. Alvarenga, A. Ravelo, et al. 1996b. Antibiotic phenol nortriterpenes from Maytenus canariensis. Phytochemistry. 43:129-132.

Gonzalez F.G., T.Y. Portela, E.J. Stipp, et al. 2001. Antiulcerogenic and analgesic effects of Maytenus aquifolium, Sorocea bomplandii and Zolernia ilicifolia. J. Ethnopharmacol. 77:41-47.

Gupta R.S., J.B. Kachhawa \& R. Chaudhary. 2004. Antifertility effects of methanolic pod extract of Albizzialebbeck (L.) Benth in male rats. Asian J. Androl. 6:155-9.

Gupta R.S. \& R. Sharma. 2006. A review on medicinal plants exhibiting antifertility activity in males. Nat. Prod. Radiance. 5:389-410.

Jorge R.M., J.P. Leite, A.B. Oliveira, et al. 2004. Evaluation of anticonceptive, anti-inflammatory and anti ulcerogenic activities of Maytenus ilicifolia. J. Ethnopharmacol. 94:93-100.

Kloucek P., B. Svobodova, Z. Polesny, et al. 2007. Antimicrobial activity of some medicinal barks used in Peruvian Amazon. J. Ethnopharmacol. 111:427-429.

Lohiya N.K., B. Manivannan, P.K. Mishra, et al. 2002. Chloroform extract of Carica papaya seeds induces long-term reversible azoospermia in langur monkey. Asian J. Androl. 4:17-26.

Madrigal R.V., B.W. Zilkowski, Jr C.R. Smith. 1985. Structure-activity relationships among maytansinoids in their effect on the European corn borer, Ostrinia nubilalis (Hubner). J. Chem. Ecol. 11:501-506.

Matu E.N. \& J. Van Staden. 2003. Antibacterial and anti-inflammatory activities of some plants used for medicinal purposes in Kenya. J. Ethnopharmacol. 87:35-41.

Medeiros R., M.F. Otuki, M.C. Avellar \& J.B. Calixto. 2007. Mechanisms underlying the inhibitory actions of the pentacyclictriterpene alpha-amyrin in the mouse skin inflammation induced by phorbol ester 12-O-tetradecanoylphorbol-13-acetate. Eur J Pharmacol. 559:227-235.

Mejia K. \& E. Reng. 1995. Usos Medicinales de Plantas de uso popular en la Amazonía Peruana. AECI y IIAP. Lima, Perú.

Montanari T., J. De Carvalho \& H. Dolder. 1998. Effect of Maytenus ilicifolia Mart. ex. Reiss on spermatogenesis. Contraception. 57:335-339.

Montanari T. \& E. Bevilacqua. 2002. Effect of Maytenus ilicifolia Mart on pregnant mice. Contraception. 65:171-175.

Nikiema J.B., R. Vanhaelen-Fastre, M. Vanhaelen, et al. 2001. Effects of antiinflammatory triterpenos isolated from Leptadeniahastata latex on keratinocyte proliferation. Phytoteraphy Research. 15:131-134.

Otake T., H. Mori, M. Morimoto, et al. 1995. Screening of Indonesian plant extracts for anti-human immunodeficiency virus type 1 (HIV-1) activity. Phytother. Res. 9:6-10.

Safavhi H., E.R. Sailer. 1997. Antiinflammatory actions of penta cyclic triterpenes. Planta Medica. 63:487-493.

Santos V.L., V.B. Costa, M.F. Agra, et al. 2007. Pharmacological studies of ethanolic extracts of Maytenus rigida Mart (Celastraceae) in animal models. Rev. Bras. Farmacogn. 17:336-342.

Vellosa J.C.R., N.M. Khalil, V.A.F. Formenton, et al. 2006. Antioxidant activity of Maytenus ilicifolia root bark. Fitoterapia.77:243-244.

White D., E. de LaMirande \& C. Gagnon. 2007. Protein kinase C is an important signaling mediator associated with motility of intact sea urchin spermatozoa. J. Exp. Biol. 210:4053-4064.

World Health Organization (WHO). Standard procedures. 2010. In: T.G. Cooper, Eds. Laboratory manual for the Examination and processing of human semen, 5 th edn. Switzerland. 